- Case Report

\title{
Mammary Paget's Disease of the Nipple: Relatively Common but Still Unknown to Many
}

\author{
Poh Siang Ooi', Nani Draman'*, Siti Suhaila Mohd Yusoff', Wan Zainira Wan Zain², Dharmendra Ganasagaran², \\ Hui Heng Chua ${ }^{3}$ \\ 'Department of Family Medicine, School of Medical Sciences, Universiti Sains Malaysia Health Campus, Kubang Kerian, Malaysia \\ ${ }^{2}$ Department of Surgery, School of Medical Sciences, Universiti Sains Malaysia Health Campus, Kubang Kerian, Malaysia \\ ${ }^{3}$ Department of Pathology, School of Medical Sciences, Universiti Sains Malaysia Health Campus, Kubang Kerian, Malaysia
}

\begin{abstract}
Mammary Paget's disease is clinically defined as skin inflammation of the nipple area and is an adenocarcinoma of the epidermis of the nipple. The pathogenesis of mammary Paget's disease is relatively unknown; nonetheless, there are two popular theories that support the underlying carcinoma and de novo carcinogenesis. For the attending medical practitioner, mammary Paget's disease poses a diagnostic and therapeutic dilemma, especially in the absence of a clinically palpable breast mass. We report a rare case of a 48-year-old Malay woman who presented at Hospital Universiti Sains Malaysia, Kelantan, Malaysia with the symptom of skin erosion on the left nipple and unresponsiveness to multiple topical treatments. A full evaluation and assessment of the patient were conducted, and mammary Paget's disease was diagnosed.
\end{abstract}

Keywords: Breast Neoplasms; Eczema; Mammary Paget's Disease; Nipples

Received: December 28, 2017, Revised: February 18, 2018, Accepted: February 28, 2018

*Corresponding Author: Nani Draman https://orcid.org/0000-0003-4143-6065

Tel: +60-1-115184421, Fax: +60-9-767-6608, E-mail: drnani@usm.my 


\section{INTRODUCTION}

Mammary Paget's disease is an adenocarcinoma that arises within the epidermis of the nipple and/or areola of the breast. Histopathologically, it is characterized by malignant glandular epithelial cells with clear and abundant cytoplasm known as Paget cells. These cells contain mucin together with pleomorphic and hyperchromatic nuclei. They are situated within the squamous epithelium of the nipple that may extend into the areola and adjacent skin. ${ }^{1)}$ Mammary Paget's disease is associated with carcinoma of the underlying lactipherous ducts. The skin lesions progress slowly over months as scaly, fissured, or oozing erythema of the nipple and areola. Advanced lesions may appear as well-demarcated, eczema-like plaques with a pink or red hue. Although uncommon, the surface may appear slightly infiltrated and erosive, oozing, scaly or crusted. ${ }^{2)}$ In the early stages, patients often present with nipple itchiness. The nipple appears to be normal; ergo, the possibility of something sinister, such as mammary Paget's disease, might be overlooked. Eczema is the common initial diagnosis, and topical treatment is invariably the first treatment that the patient receives. Therefore, high levels of clinical suspicion and detailed physical examination is pivotal to avoid missing the diagnosis of such a disease.

The case presented is that of a 48-year-old woman without any significant risk factors for breast cancer, who presented with an abnormal-looking nipple without other associated symptoms. The patient was later diagnosed with mammary Paget's disease.

\section{CASE REPORT}

Madam K, a 48-year-old school teacher, complained of changes on her left nipple that had persisted for the last 10 months. The left nipple appeared to be more distorted and larger than the right nipple. There were no common symptoms associated with itchiness, pain, or any nipple discharge during this time. There was no palpable breast lump either. The contralateral side of the breast and nipple areolar complex appeared normal. Madam K did experience loss of appetite and significant loss of weight (from 51 to $47 \mathrm{~kg}$ ) in the past 3 months. She had not bought a new brassiere in the last year.

Prior to the tertiary hospital visit, Madam $\mathrm{K}$ had visited five general practitioners in a period of 10 months seeking treatment for her left nipple. She was told by the doctors that the changes on the nipple may be caused by inconspicuous injuries. Different topical creams were prescribed to her, but none resulted in improvement. She was even administered a course of antibiotics, which did not yield any benefits. During her visit to the different doctors, she did not disclose information pertaining to the treatment provided by the previous medical practitioner. Therefore, the medical practitioners had no knowledge of previous consultations or treatments. The information could otherwise be obtained through thorough history-taking of the patient. During the last consultation, the doctor suspected malignancy and immediately referred Madam $\mathrm{K}$ to a tertiary hospital for further evaluation and treatment.

Madam K has no significant past medical history. There was no history of atopy among herself or her family members. There was no family history of any skin disease or malignancy. Madam K attained menarche at the age of 15 years. She is currently married with two children. She gave birth to her first child at the age of 35 years. She breastfed both of her children until they were 1 year old. She was not taking any hormonal contraception. She is also a non-smoker. A full clinical examination revealed that Madam $\mathrm{K}$ is a generally healthy woman.

On inspection, the areola appeared normal, but the central skin extending to the lateral side of the left nipple was eroded (Figure 1). No nipple discharge was noted. The surrounding skin of her breast had multiple moles with no other abnormalities found. There was no concurrent palpable breast lump or axillary lymph nodes. The contralateral right breast was normal.

Based on the history and clinical assessment, Paget's disease of the breast was suspected. Thus, Madam K was immediately referred for an urgent nipple biopsy, and arrangements were made to perform a mammogram. The left breast mammography showed highly pleomorphic calcification with a casting pattern, with regional distribution in the left retro-areolar region, with no focal dominant lesion BIRADS (Breast Imaging Reporting and Data System) V (Figure 2).

The biopsy of the left nipple showed ulcerated stratified squamous epithelium infiltrated by atypical cells arranged in clusters and singly dispersed. The cells were large, with pleomorphic vesicular nuclei and prominent eosinophilic and pale cytoplasm (immunochemistry: cytokeratin-7, positive; Cerb-B2, strongly positive). This is compatible with Paget's disease of the breast. Computed tomography of the thorax, abdomen, and pelvis showed no evidence of metastatic disease, while the bone scan showed no osteoblastic disease.
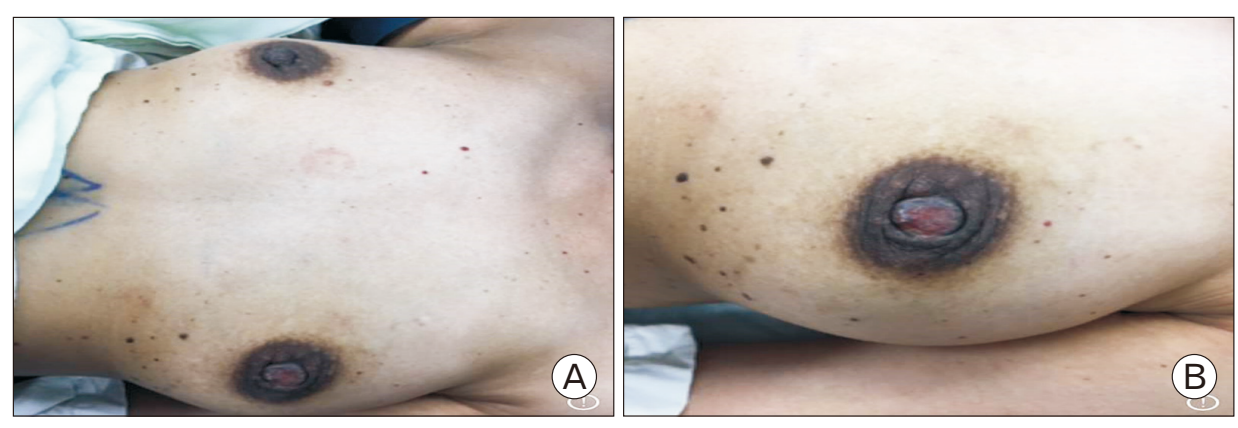

Figure 1. (A, B) The left nipple was eroded from the center, extending to the lateral side. 

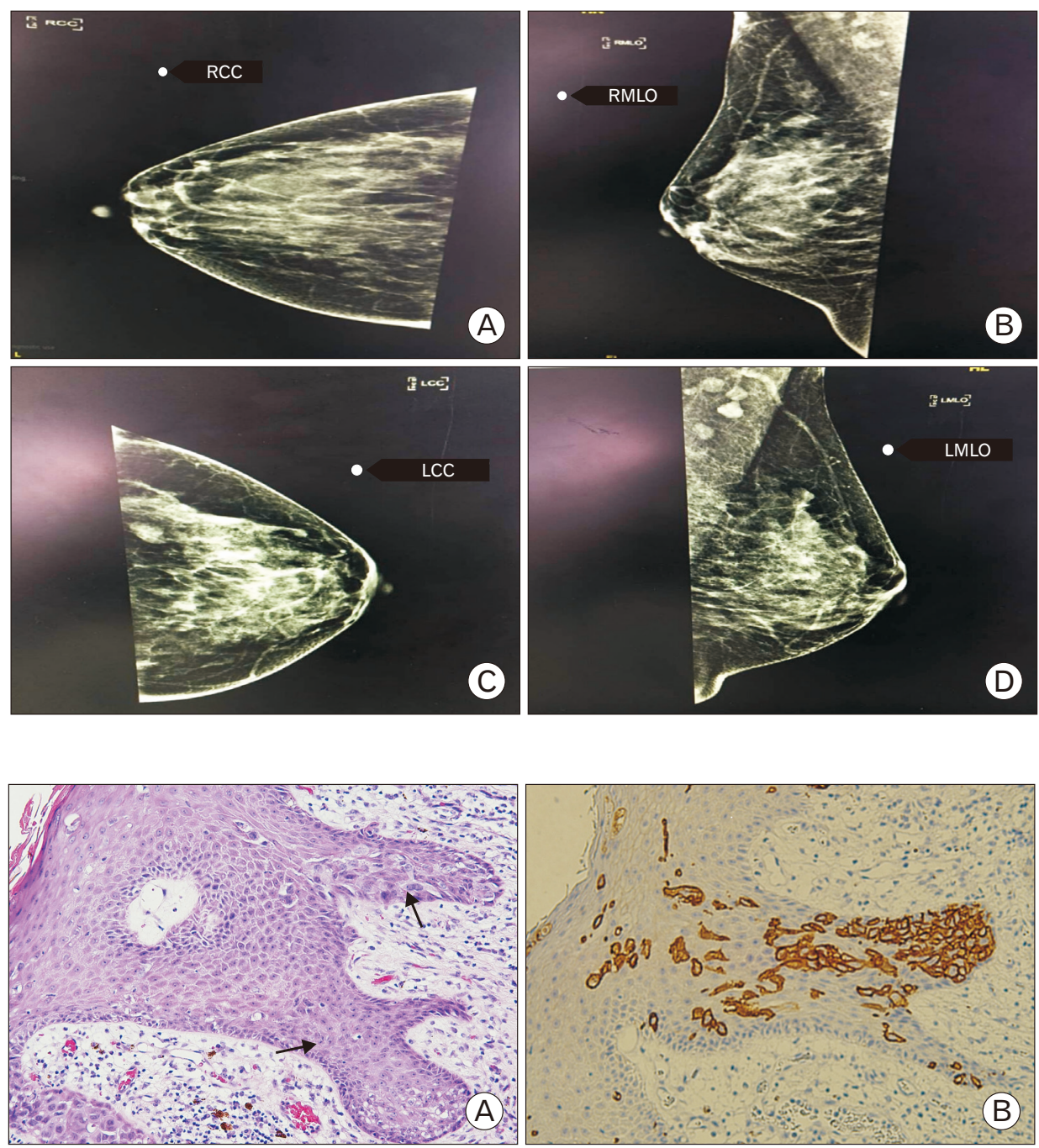

Figure 2. (A-D) Mammography of the left breast (LCC and LMLO) shows highly pleomorphic calcification with a casting pattern, with regional distribution in the left retro-areolar region with no focally dominant lesion BIRADS (Breast Imaging Reporting and Data System) V. LCC, left craniocaudal view; LMLO, left mediolateral oblique view; RCC, right craniocaudal view; RMLO, right mediolateral oblique view.

Figure 3. (A) Section shows the epidermis of the nipple infiltrated by large Paget's cells, which are singly dispersed exhibiting pleomorphic vesicular nuclei with prominent nucleoli and pale cytoplasm (×200, arrows). (B) The tumor cells were positive for c-erbB-2 immunohistochemical staining $(\times 200)$.
Madam K underwent left mastectomy and axillary clearance with immediate left breast reconstruction with pedicled TRAM (transverse rectus abdominis musculocutaneous) flap on August 22, 2017. On histopathology examination, the mastectomy specimen showed that the epidermis was infiltrated by single large pleomorphic cells exhibiting large vesicular nuclei with prominent nucleoli. The cytoplasm was pale and abundant. A section from the breast tissue show foci of highgrade ductal carcinoma in situ (DCIS) exhibiting comedo necrosis and occasional cribriform pattern. There were eight lymph nodes that exhibited reactive lymphoid hyperplasia. All surgical margins were free from the DCIS component (immunohistochemistry: estrogen and progesterone receptors, both negative; c-erbB-2, 3+). The conclusion was high-grade DCIS with Paget's disease of the nipple (Figure 3A, B). A written informed consent had been taken from Madam $\mathrm{K}$ for publication of her case.

\section{DISCUSSION}

Mammary Paget's disease is a known cause of eczematous lesions of the nipple with an incidence of $1 \%$ of all breast cancers. Most cases are affiliated with an underlying neoplasm ranging from DCIS to invasive cancers. Paget's breast disease is characterized histologically by an epidermal migration and intraductal spread of Paget's cells. It was demonstrated that the outcome of patients with Paget's disease of the breast could be determined by the presence or absence of invasive cancer in the underlying breast tissue. ${ }^{3)}$ In Malaysia, according to the national cancer registry, there were only eight cases reported of 18,258 breast cancer cases from 2007 to 2011 . $^{4)}$ The prevalence is slightly higher at $0.7 \%-4.3 \%$ in other parts of the world. ${ }^{2)}$

Mammary Paget's disease exhibits a gradual onset that evolves over months to years and usually affects the breast unilaterally. The disease starts from the nipple area and gradually extends to the areola and to the surrounding skin in more advanced cases. The lesions appear eczematoid, erythematous, thickened, moist, or crusted with irregular borders, with or without fine scaling, induration, infiltration, secretion, bleeding, ulceration, and nipple invagination. ${ }^{5)}$ Usually, this disease is more prevalent among those who are post-menopausal or in their sixties. ${ }^{5)}$ However, the patient in this case is 48 years old and not meno- 
pausal.

Due to the wide range of presentation of this disease, it is common for patients presenting with these features to be misdiagnosed and to be treated for eczema, psoriasis, melanoma, infection, or trauma. ${ }^{5-7)}$ For example, a patient may be treated for eczema with topical steroids initially with hope for recovery. If there was minimal improvement or persistent nipple abnormality with optimal treatment for eczema, the medical practitioner should be notified and patient should be referred for a biopsy to avoid undue delay in a definitive diagnosis.

It is crucial for patients to provide accurate histories and inform the attending doctor of previous treatments for an effective overall view of the progression of the disease before deciding on the next appropriate management plan. Another method to recognize the early changes in mammary Paget's disease is by using a dermoscope. A brief report has been published regarding the use of a dermoscope to visualize the vascularity and thus aid the clinician in investigating the suspicion of such conditions over other differential diagnoses, such as eczema. ${ }^{8)}$

In this case, the failure to obtain an accurate diagnosis for months resulted in a more locally or loco-regionally advanced disease diagnosis. Madam K was diagnosed with high-grade DCIS with Paget's disease of the nipple with suspected spinal metastasis.

Routine mammography is performed in suspected cases of Paget's disease to identify microcalcifications, heterogeneous and suspicious opacities. However these findings might be difficult to be detected. In the presence of a palpable mass, the sensitivity of mammography to detect a tumor is $97 \%$, whereas it is only $50 \%$ when no mass is palpable clinically. ${ }^{9)}$ Paget's disease without a palpable breast tumor is clinically non-aggressive and can be managed well by breast-conserving therapy, such as local excision and/or irradiation. ${ }^{10)}$

Mammary Paget's disease should be one of the diseases suspected in cases of persistent eczematous involvement of the nipple that remain non-responsive to topical treatment. The prognosis of mammary Paget's disease depends on the presence or absence of an underlying carcinoma and the stage of the disease. It was reported that the 5 -year recurrence-free survival is $75 \%-90 \%$ for those with DCIS and $63 \%-75 \%$ for those with invasive carcinoma. Meanwhile, the 5-year overall survival rates are $94 \%-98 \%$ and $73 \%-93 \%$ in the presence of DCIS and invasive carcinoma, respectively. ${ }^{1)}$

\section{CONFLICT OF INTEREST}

No potential conflict of interest relevant to this article was reported.

\section{ORCID}

Poh Siang Ooi: https://orcid.org/0000-0003-2612-1663

Nani Draman: https://orcid.org/0000-0003-4143-6065

Siti Suhaila Mohd Yusoff: https://orcid.org/0000-0003-1149-5531

Wan Zainira Wan Zain: https://orcid.org/0000-0001-8019-6063

Dharmendra Ganasagaran: https://orcid.org/0000-0002-9649-5778

Hui Heng Chua: https://orcid.org/0000-0002-1954-4476

\section{REFERENCES}

1. Lakhani SR, Ellis IO, Schnitt SJ, Tan PH, van de Vijver MJ. WHO classification of tumours of the breast. 4th ed. Lyon: International Agency for Research on Cancer; 2012.

2. Kanitakis J. Mammary and extramammary Paget's disease. J Eur Acad Dermatol Venereol 2007;21:581-90.

3. Paget J. On disease of the mammary areola preceding cancer of the mammary gland. St Bartholomew's Hosp Rep 1874;10:87-9.

4. National Cancer Institute, Ministry of Health. The Malaysian National Cancer Registry report 2007-2011: Malaysia cancer statistics, data and figures. Putrajaya: National Cancer Institute, Ministry of Health Malaysia; 2015.

5. Lopes Filho LL, Lopes IM, Lopes LR, Enokihara MM, Michalany AO, Matsunaga N. Mammary and extramammary Paget's disease. An Bras Dermatol 2015;90:225-31.

6. Brugues A, Iranzo P, Diaz A, Pena A, Estrach MT, Carrera C. Pigmented mammary Paget disease mimicking cutaneous malignant melanoma. J Am Acad Dermatol 2015;72:e97-8.

7. Liu J, Zhao Z, Zhou J, Zhang J. An unusual presentation of Paget's disease of the nipple in a young woman: a case report. Int J Clin Exp Med 2015;8:4694-6.

8. Errichetti E, Avellini C, Pegolo E, de Francesco V. Dermoscopy as a supportive instrument in the early recognition of erosive adenomatosis of the nipple and mammary Paget's disease. Ann Dermatol 2017; 29:365-7.

9. Karamchandani DM, Chetlen AL, Riley MP, Schetter S, Hollenbeak CS, Mack J. Pathologic-radiologic correlation in evaluation of retroareolar margin in nipple-sparing mastectomy. Virchows Arch 2015;466:27987.

10. Marshall JK, Griffith KA, Haffty BG, Solin LJ, Vicini FA, McCormick B, et al. Conservative management of Paget disease of the breast with radiotherapy: 10- and 15-year results. Cancer 2003;97:2142-9. 